\title{
Strategies for the Synthesis of Graphene, Graphene Nanoribbons, Nanoscrolls and Related Materials
}

\author{
Urmimala Maitra, H. S. S. Ramakrishna Matte, Prashant Kumar, and C. N. R. Rao*
}

\begin{abstract}
Single-layer graphene (SLG), the $3.4 \AA$ thick two-dimensional sheet of $\mathrm{sp}^{2}$ carbon atoms, was first prepared in 2004 by mechanical exfoliation of graphite crystals using the scotch tape technique. Since then, SLG has been prepared by other physical methods such as laser irradiation or ultrasonication of graphite in liquid media. Chemical methods of synthesis of SLG are more commonly used; the most popular involves preparation of single-layer graphene oxide followed by reduction with a stable reagent, often assisted by microwave heating. This method yields single-layer reduced graphene oxide. Other methods for preparing SLG include chemical vapour deposition over surfaces of transition metals such as Ni and Cu. Large-area SLG has also been prepared by epitaxial growth over SiC. Few-layer graphene (FLG) is prepared by several methods; arc discharge of graphite in hydrogen atmosphere being the most convenient. Several other methods for preparing FLG include exfoliation of graphite oxide by rapid heating, ultrasonication or laser irradiation of graphite in liquid media, reduction of few-layer graphene oxide, alkali metal intercalation followed by exfoliation. Graphene nanoribbons, which are rectangular strips of graphene, are best prepared by the unzipping of carbon nanotubes by chemical oxidation or laser irradiation. Many graphene analogues of inorganic materials such as $\mathrm{MoS}_{2}, \mathrm{MoSe}_{2}$ and $\mathrm{BN}$ have been prepared by mechanical exfoliation, ultrasonication and by chemical methods involving high-temperature or hydrothermal reactions and intercalation of alkali metals followed by exfoliation. Scrolls of graphene are prepared by potassium intercalation in graphite or by microwave irradiation of graphite immersed in liquid nitrogen.
\end{abstract}

Keywords: Graphene
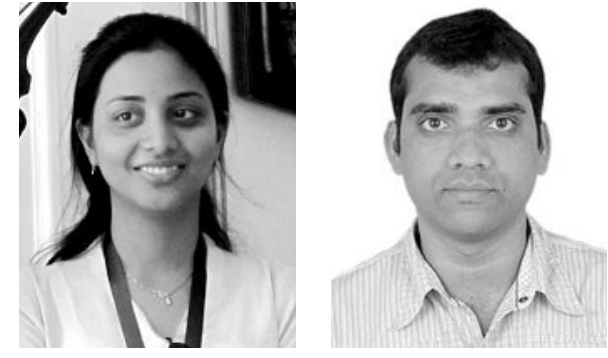

Urmimala Maitra (left) obtained her BSc degree from Banaras Hindu University in 2007 and MS degree in Materials Science in 2010 from Jawaharlal Nehru Centre for Advanced Scientific Research. She is presently doing her PhD work on nanomaterials.

H. S. S. Ramakrishna Matte (right) obtained his BSc degree from Government College (Auto-nomous), Rajahmundry in 2006 and MS degree in chemical science in 2009 from the Jawaharlal Nehru Centre for Advanced Scientific Research. He is completing his PhD work related to synthesis, characterization and properties of graphene and its inorganic analogues.

\footnotetext{
${ }^{*}$ Correspondence: Prof. C. N. R. Rao Chemistry and Physics of Materials Unit CSIR Centre of Excellence

International Centre for Materials Research Jawaharlal Nehru Centre for Advanced Scientific Research

Jakkur P. O., Bangalore, 560064, India

E-mail: cnrrao@jncasr.ac.in
}
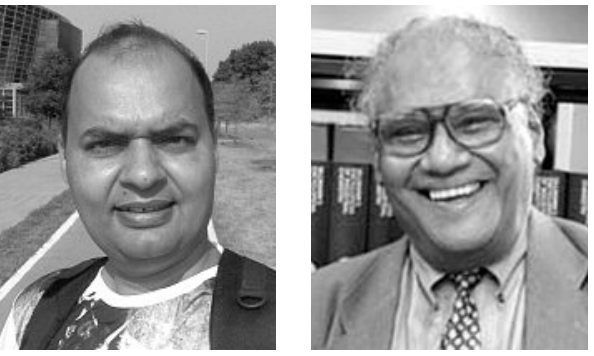

Prashant Kumar (left) got his doctoral degree in Physics in 2009 from University of Hyderabad and has worked for three years at JNCASR Bangalore with Prof. C. N. R. Rao as Nanoscience and Technology Post Doctoral Fellow under DST's Nano Initiative Program of Govt. of India. Now he has moved to Birck Nanotechnology Centre of Purdue University, West Lafayette, Indiana, USA. His research broadly pertains to understanding and exploiting the interactions of matter with exotic fields such as extreme electric field and excimer laser for nanotechnology applications including the synthesis of novel materials of reduced dimensionalities such as graphene and its inorganic analogues.

C. N. R. Rao (right) obtained his $\mathrm{PhD}$ degree from Purdue University (1958) and DSc degree from the University of Mysore (1961). He is the National Research Professor and Linus Pauling Research Professor at the Jawaharlal Nehru Centre for Advanced Scientific Research and
Honorary Professor at the Indian Institute of Science (both at Bangalore). His research interests are mainly in the chemistry of materials. He is a member of many of the science academies and an honorary fellow of RSC. He is the recipient of the Einstein Gold Medal of UNESCO, the Hughes and Royal Medals of the Royal Society, the August Wilhelm von Hofmann medal of the German Chemical Society, the Dan David Prize for materials research and the first India Science Prize.

\section{Introduction}

Although graphene has been a subject of interest for several decades, its isolation became possible only recently, by simple micromechanical exfoliation of graphite flakes using scotch tape. ${ }^{[1]}$ After this discovery in 2004, several methods have been employed for the preparation of singleand few-layer graphenes. These methods include both physical and chemical approaches. Other than the micromechanical method, the other physical methods for the synthesis of single-layer graphene (SLG) include ultrasonication of dispersion of graphite in liquids, and laser irradiation. ${ }^{[2]}$ Many of the strategies for the synthesis of SLG involve oxidation of graphite to graphite oxide (GO) followed by exfoliation and reduction. Sonochemical exfolia- 
tion of GO yields single-layer graphene oxide (SGO) which on chemical reduction gives reduced graphene oxide (RGO). This is the most commonly used method.[3] Exfoliation of alkali metal ion intercalated graphite also yields SLG. ${ }^{[4]}$ Other chemical methods are chemical vapour deposition (CVD) over transition metal surfaces and epitaxial growth on $\mathrm{SiC}$ substrate. ${ }^{[2 b]}$ Several methods have been employed for the synthesis of few-layer graphene (FLG) containing 2-10 layers. Thus, thermal exfoliation and reduction of GO by rapid heating above $1000^{\circ} \mathrm{C}$ yields FLG. ${ }^{[5]}$ Other physical methods include arc discharge of graphite in hydrogen atmosphere (HG) and conversion of diamond to graphene in a helium (DG). ${ }^{[6]}$ Pyrolysis of aerosols and laser-induced reduction of graphene oxide have also been carried out to obtain FLG.

In this article, we briefly present the various strategies to prepare single- and few-layer graphene by both physical and chemical methods. We then examine the synthesis of graphene nanoribbons (GNR) which have unique electronic and magnetic properties. The most commonly used method for the synthesis of GNRs involves unzipping carbon nanotubes by oxidation. ${ }^{[7]}$ Recently, unzipping of nanotubes has been achieved using laser irradiation. ${ }^{[8]}$ Synthesis of graphene nanoscrolls derived from graphene or graphite by rolling up of a single sheet or a few sheets of graphene will also be discussed. The synthesis of graphene analogues of layered inorganic materials such as boron nitride and layered transition metal chalcogenides will be briefly outlined.

\section{Single-layer Graphene}

Single-layer graphene, the $3.4 \AA$ thick two-dimensional sheet of $\mathrm{sp}^{2}$ carbon atoms, is transparent. It is readily characterized by atomic force microscopy (AFM), Raman spectroscopy and other techniques. Single-layer graphene cannot be stored as a solid since it converts to graphite. It is, therefore, necessary to deposit SLG on a solid substrate or keep it as a dispersion in solvent.

\subsection{Physical Methods}

\subsubsection{Micromechanical Exfoliation}

Overlap of $\mathrm{p}_{\mathrm{z}}$ or $\pi$ orbitals perpendicular to the plane of the graphene sheet (via van der Waals interaction) leads to the stacking of sheets in graphite. Graphite therefore has weak bonding in the direction perpendicular to the plane of the sheets. Exfoliation is the reverse of stacking involving peeling off of the layers from graphite. Single-layer graphene was obtained by Noveselov et al. .1,9] $^{[1}$ sing scotch tape to peel off graphene layers from highly oriented pyrolytic graphite (HOPG). They pressed patterned HOPG square meshes on a photoresist spun over a glass substrate and peeled off the layers using scotch tape. The scotch tape method was later modified to peel off few layers of graphene and deposit on a solid substrate such as a $\mathrm{SiO}_{2}(300 \mathrm{~nm}) / \mathrm{Si}$. Manipulation of graphene sheets have been achieved using AFM and STM tips. ${ }^{[10]}$ The degree of folding of the sheets can be modulated by changing the bias voltage and the distance between the tip and the sample. ${ }^{[10 f]}$

Optical contrast of single-, bi- and fewlayer graphene is different on different substrates arising from interference of the reflected light beam at the air-to-graphene, graphene-to-dielectric and dielectric-tosubstrate interfaces. ${ }^{[11]}$ Fig. 1a shows the optical image of one-, two-, three- and four-layer graphene on $\mathrm{Si}$ substrates with $285 \mathrm{~nm} \mathrm{SiO}$. The thickness of graphene layers is directly probed by atomic force microscopy (AFM) in the tapping mode and the number of layers can be directly calculated from interlayer distance in graphite. ${ }^{[12]}$ Fig. 1b shows the AFM image of one-, two- and five- layered graphene on $\mathrm{SiO}_{2} / \mathrm{Si}$ substrate.[1] Scanning tunneling microscopy (STM) and transmission electron microscopy (TEM) provide high resolution images of graphene. Raman spectroscopy is a non-destructive tool that provides valuable information about the structural and electronic characteristics of graphene. ${ }^{[12]}$ Fig. 1c shows typical Raman spectra of one-, two-, three- and four-layered graphene. The Raman spectrum of graphene consists of three major bands the defect-induced D-band $\left(1300 \mathrm{~cm}^{-1}\right)$ band,
G-band $\left(1580 \mathrm{~cm}^{-1}\right)$ due to the in-plane vibrations of the $\mathrm{sp}^{2}$ carbon atoms and the 2D band $\left(2700 \mathrm{~cm}^{-1}\right)$ that results from a second-order process.[13] The 2D band broadens with increasing number of layers as shown in Fig. 1d. ${ }^{[14]}$

\subsubsection{Exfoliation in Solvents}

Single-layer graphene can be obtained by solvent-phase exfoliation of graphite in solvents like N-methylpyrrolidone (NMP). ${ }^{[15]}$ The energy required to exfoliate graphene must be comparable to the solvent-graphene interaction for exfoliation to occur. The energy required to initiate exfoliation is provided sonochemically. Dispersion of graphene with concentrations up to $0.01 \mathrm{mg} \mathrm{ml}^{-1}$ are obtained by this method. Fig. 2a shows the dispersion of graphene in NMP with decreasing concentrations of graphene (A to E). Graphene thus obtained is mostly singleand bi-layer. Graphene can be dispersed in perfluorinated aromatic solvents, ${ }^{[16]}$ orthodichlorobenzene ${ }^{[17]}$ and even in low-boiling solvents such as chloroform and isopropanol. ${ }^{[18]}$ A TEM image of a large area single-layer graphene is shown in Fig. $2 b$. The inset shows the enlarged view of the highlighted region where the sheet is folded to reveal it is indeed made up of monolayer. ${ }^{[17]}$ Hernandez et al. ${ }^{[19]}$ have shown that good solvents for graphene can be characterized by Hildebrand and Hansen solubility parameters.

Laser irradiation of graphite powder in a polar medium such as dimethylformamide (DMF) readily gives rise to singleas well as few-layer graphenes. A dispersion of graphite in DMF when irradiated by a Lambda Physik KrF excimer laser $(\gamma$

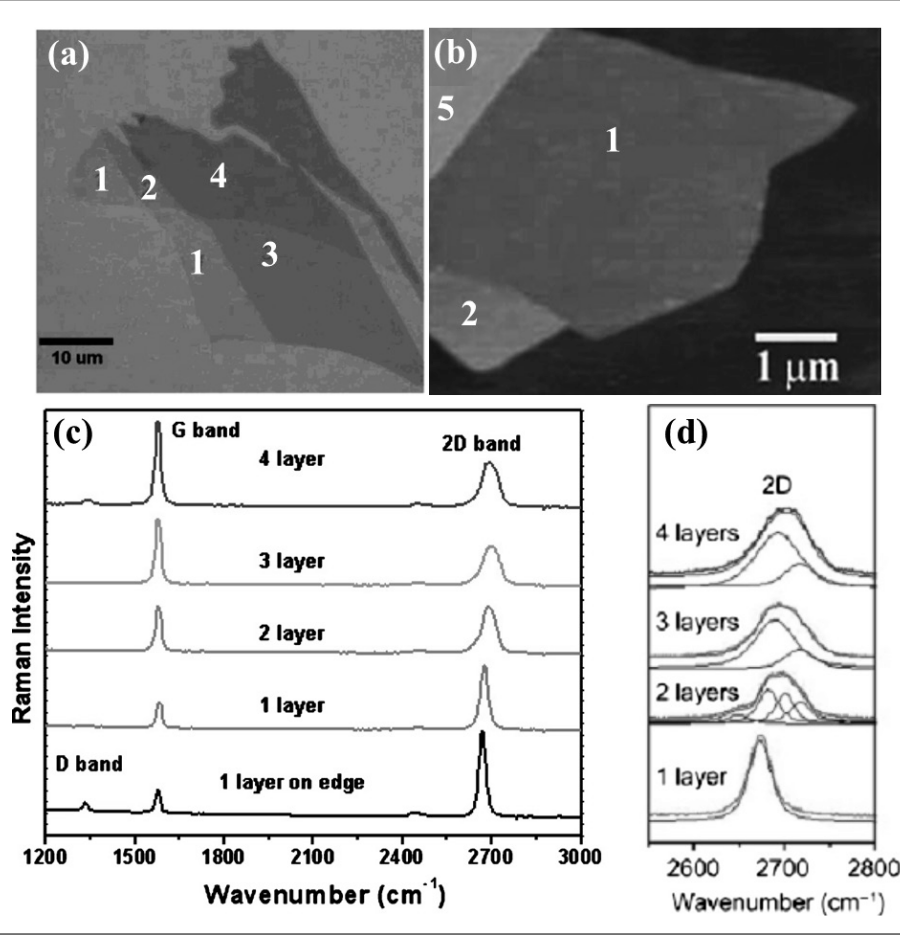

Fig. 1. (a) Optical image of one-, two-, three- and fourlayered graphene on $\mathrm{SiO}_{2}(285 \mathrm{~nm}) / \mathrm{Si}$ substrate (reprinted with permission from ref. [14]. Copyright American Chemical Society, 2007), (b) AFM image of graphene sheet with thickness $0.8 \mathrm{~nm}$ (one-layer), $1.2 \mathrm{~nm}$ (two-layers) and 2.5 $\mathrm{nm}$ (five-layers) (modified from ref. [1]), (c) Raman spectra as a function of number of layers and (d) the zoom in view of the 2D band (reprinted with permission from ref. [14]. Copyright American Chemical Society, 2007). 


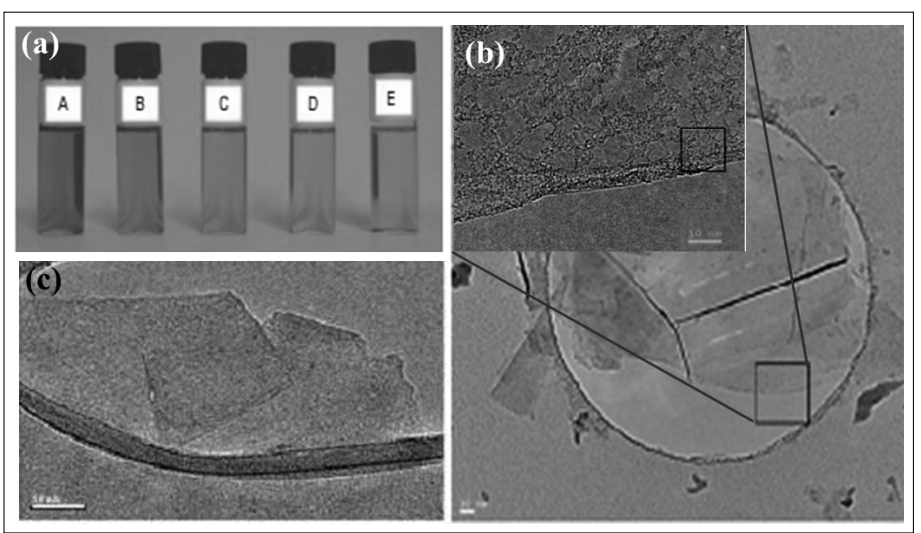

Fig. 2. (a) Dispersions of graphite flakes in NMP, at a range of concentrations ranging from $6 \mu \mathrm{g} / \mathrm{ml}(A)$ to $4 \mu \mathrm{g} / \mathrm{ml}(\mathrm{D})$ and $(E)$ after centrifugation (reprinted with permission from ref. [15]. Copyright Nature Publishing Group, 2008), (b) TEM image of graphene obtained by solvent-phase exfoliation (reprinted with permission from ref. [17]. Copyright American Chemical Society, 2009). Inset shows the selected region, and (c) TEM image of ultrathin graphene obtained by laser exfoliation of graphite in DMF.

\section{$=248 \mathrm{~nm}, \tau=30 \mathrm{~ns}$ ) at a fluence of $1.5 \mathrm{~J} /$} $\mathrm{cm}^{2}$ and $5 \mathrm{~Hz}$ repetition rate for 1 hour followed by centrifugation results in a mixture of single- and few-layer graphenes. TEM image of the supernatant liquid in Fig. 2c shows the presence of an ultra-thin graphene flake.

\subsection{Chemical Methods}

Though mechanical and sconochemical exfoliation produces graphene of the highest quality (with least defects), the method is limited by low productivity. Chemical exfoliation, on the other hand, possesses advantages of bulk scale production.

\subsubsection{Reduction of Graphene Oxide}

Ultrasonication of graphite oxide in solution yields single-layer graphene oxide (SGO) ${ }^{[3]}$ The interlayer spacing increases from $3.7 \AA$ in graphite to $9.5 \AA$ in GO making it easier to carry out exfoliation by mild sonication in solvents. Strong oxidizing agents like $\mathrm{KMnO}_{4}$ and $\mathrm{NaNO}_{3}$ in $\mathrm{H}_{2} \mathrm{SO}_{4} /$ $\mathrm{H}_{3} \mathrm{PO}_{4}$ are used to prepare $\mathrm{GO}$ by the standard Hummers method..$^{[3 a-c]}$ Single-layer reduced graphene oxide (RGO) is prepared through chemical, thermal, or electrochemical reduction. ${ }^{[20]}$ Most strong reducing agents such as $\mathrm{NaBH}_{4}$ strongly react with water while weak reducing agents like hydrazine monohydrate do not. Chemical reduction of SGO using hydrazine hydrate gives well dispersed single layer graphene sheets. ${ }^{[3 b, e, 21,22]}$ Other reducing agents used are phenyl hydrazine, ${ }^{[23]}$ hydroxylamine, ${ }^{[24]}$ glucose, ${ }^{[25]}$ ascorbic acid,,26] hydroquinone, ${ }^{[27]}$ alkaline solutions, ${ }^{[28]}$ pyrrole ${ }^{[29]}$ etc. Fig. 3a shows an AFM image of typical single layer RGO sheets. The conductivity of RGO is considerably higher $\left(10^{8}\right.$ times $)$ than that of graphene oxide. The Raman spectrum of RGO shows a strong D band due to the presence of hydroxyl and other groups on the surface. Single-layered RGO exhibits a very weak $2 \mathrm{D}$ band compared to the strong 2D band in mechanically exfoliated single-layer graphene. Fig. 3b compares the Raman band intensities of $\mathrm{D}, \mathrm{G}$, and 2D bands for mechanically exfoliated graphene with RGO.

Oxygen functionalities in SGO can also be minimized by irradiation with ultraviolet radiation or excimer laser. Solution of SGO when irradiated with light from mercury lamp $\left(254 \mathrm{~nm}, 25 \mathrm{~W}, 90 \mu \mathrm{W} / \mathrm{cm}^{2}\right)$ shows considerable decrease in oxygen containing functional groups like carbonyl. ${ }^{[30]}$ Laser irradiation of SGO solution with a $\mathrm{KrF}$ excimer laser $(248 \mathrm{~nm}, 5 \mathrm{~Hz})$ for $2 \mathrm{~h}$ also shows similar effects. This technique has been successfully used to laser pattern graphene sheets onto substrates with a TEM grid as the mask. A schematic of the process of laser reduction is shown in Fig. 4a. Fig. 4b shows the optical image of the pattern obtained after reduction with excimer laser. ${ }^{[30]}$ E-beam induced pat-

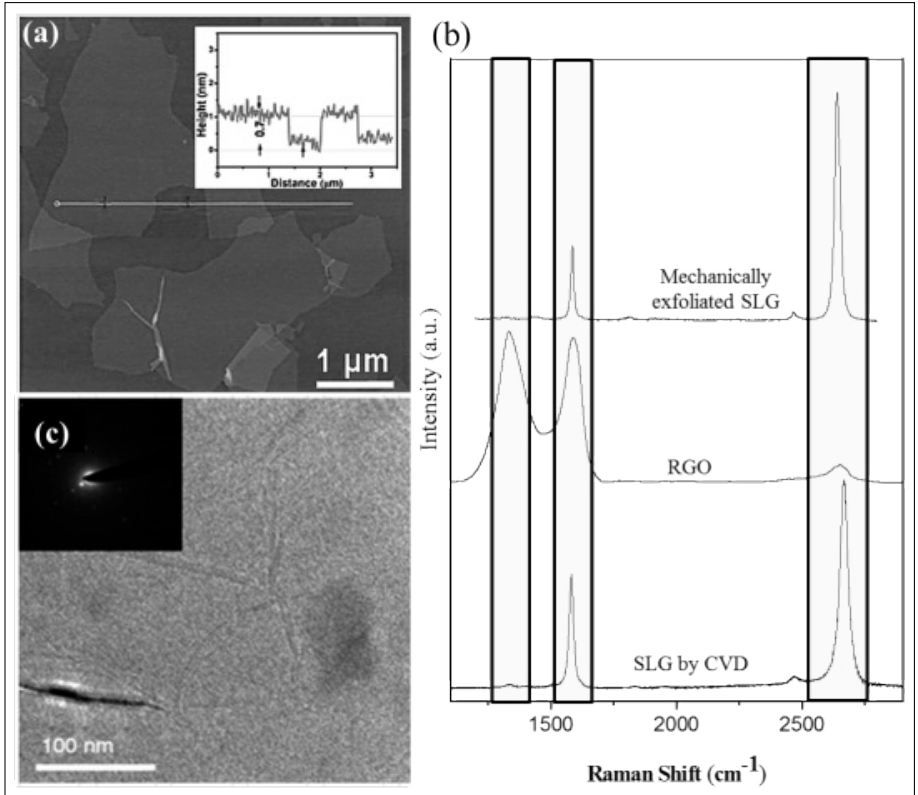

Fig. 3. (a) AFM image and height profile of RGO (reprinted with permission from ref. [24]. Copyright American Chemical Society, 2011), (b) Raman spectrum of SLG obtained from micromechanical cleavage, reduction of GO and CVD over Ni surface. Green, blue and the orange regions show the $\mathrm{D}, \mathrm{G}$ and $2 \mathrm{D}$ band positions respectively and (c) TEM image of single layer graphene obtained from methane over Ni surface (reprinted with permission from ref. [2b]. Copyright IOP Publishing, 2010).

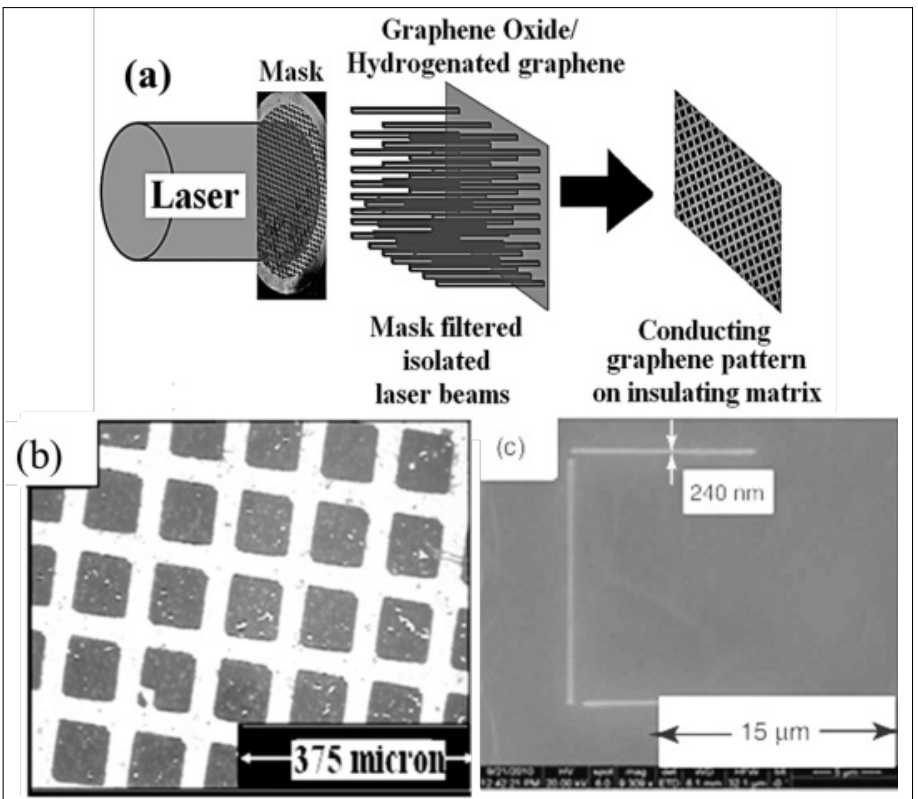

Fig. 4. (a) Schematic diagram illustrating masked laser patterning, (b) Optical microscopic image of the pattern achieved after excimer laser reduction of graphene oxide ${ }^{[30]}$ and (c) electron-beam pattern with $240 \mathrm{~nm}$ wide lines of RGO on GO films (reprinted with permission from ref. [31]). 
terning of graphene oxide films yield RGO patterns as thin as $240 \mathrm{~nm}$ (see Fig. 4c). ${ }^{[31]}$

\subsubsection{Chemical Vapour Deposition}

Chemical vapour deposition yields high quality single-layer graphene. The method is not only inexpensive but also readily accessible. Transition metal substrates such as $\mathrm{Ni},{ }^{[32]} \mathrm{Pd},{ }^{[33]} \mathrm{Ru},{ }^{[34]} \mathrm{Ir}^{[35]}$ or $\mathrm{Cu}^{[36]}$ are used as substrates to deposit graphene. Upon exposure to hydrocarbons at high temperatures the transition metal surface becomes saturated with $\mathrm{C}$. On cooling under inert or $\mathrm{H}_{2}$ atmosphere, the solubility of the carbon in the substrate decreases and a thin film precipitates on the surface. ${ }^{[37]}$ Different hydrocarbons such as methane, ethylene, acetylene and benzene have been decomposed on various transition metal substrates like $\mathrm{Ni}, \mathrm{Cu}, \mathrm{Co}, \mathrm{Au}$ and $\mathrm{Ru} .^{[2 b]}$ The number of layers varies with the hydrocarbon, transition metal and the reaction parameters such as flow rate and temperature. Fig. 3c shows a large flake of SLG prepared on a Ni substrate with methane at $1000{ }^{\circ} \mathrm{C}$. Graphene prepared by this method is highly pure. The Raman spectrum of CVD graphene shows an almost inconspicuous D band, sharp $G$ and 2D bands (see Fig. 3b). [2b] The 2D band, the key feature in mechanically exfoliated SLG, is equally prominent in CVD graphene. The graphene can be transferred to other substrates by etching of the underlying transition metal. Radio frequency plasma enhanced chemical vapour deposition (PECVD) system has also been used to synthesize graphene on various substrates such as $\mathrm{Si}$, W, Mo, Zr, Ti, Hf, Nb, Ta, Cr, stainless steel, $\mathrm{SiO}_{2}$ and $\mathrm{Al}_{2} \mathrm{O}_{3} \cdot{ }^{[38]}$

Gram quantities of single layer graphene have been produced by the pyrolysis of ethanol trapped in metal alkoxides. ${ }^{[39]}$ An alcoholic solution of the metal under solvothermal conditions entraps free ethanol in a clathrate-like structure in the metal-alkoxide. This is then pyrolized to yield a fused array of graphene sheets, and sonicated to obtain single-layer graphene. Ternary potassium salt $\mathrm{K}(\mathrm{THF})_{\mathrm{x}} \mathrm{C}_{24}$ are used to intercalate potassium into graph-
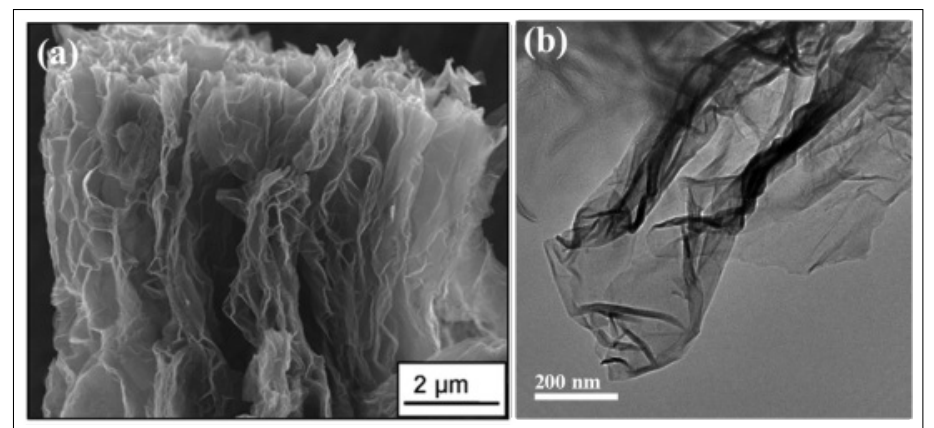

Fig. 5. (a) FESEM and (b) TEM images of graphene obtained from thermal exfoliation of graphite oxide. Reprinted with permission from Rao et al.,

Nanotechnology 2009, 20, 125705. Copyright IOP Publishing, 2009. ite. K-intercalated graphite readily exfoliates in NMP without any vigorous reaction to yield a dispersion of negatively charged single-layer graphene.[4] Noncovalent functionalization and solubilization of graphene in water along with direct exfoliation is possible by employing the potassium salt of coronene tetracarboxylic acid (CS) to yield monolayer graphene-CS composites. ${ }^{[40]}$

\section{Few-layer Graphenes}

For many applications, few-layer graphene samples with two or three layers may suffice. Few-layer graphenes are easily prepared and can also be stored.

\subsection{Physical Method}

\subsubsection{Thermal Exfoliation of GO}

Graphite oxide undergoes thermal expansion and exfoliation into individual sheets by rapid heating $\left(>200{ }^{\circ} \mathrm{C} /\right.$ min) to $\sim 1000{ }^{\circ} \mathrm{C}$ through evolution of $\mathrm{CO}_{2}{ }^{[5]}$ Because of rapid heating, the decomposition rate of epoxy and hydroxyl groups exceeds the rate of diffusion of the evolved gases like $\mathrm{CO}_{2}$ and $\mathrm{H}_{2} \mathrm{O}$ generating pressures enough to overcome the van der Waals forces holding the graphene sheets together. Graphene sheets exfoliated in this manner (exfoliated graphene or EG) are generally wrinkled and possess defects. Because of wrinkling, the sheets do not collapse and restack back to graphite and remain as agglomerated lumps of few-layered graphene. A FESEM image of such a lump of EG is shown in Fig. 5a. The wrinkled nature of the graphene is clearly seen. The TEM image in Fig. 5b shows a large sheet of few-layer thick graphene. Commercial expandable graphite also undergoes exfoliation by heating at $1000{ }^{\circ} \mathrm{C}$ in forming gas for 60 secs. ${ }^{[41]}$ Acid-intercalated graphite oxide undergoes exfoliation in microwave. ${ }^{[42]}$ Eddy currents are generated when acidintercalated graphite is irradiated with microwave because of the stratified structure of GO, yielding very high temperatures in a short time by Joules heating. This thermal shock leads to sudden decomposition and gasification of the intercalated acids in graphite. The pressure thus created breaks the interlayer van der Walls forces.

Graphene oxide can be reversibly reduced and oxidized as demonstrated by Ekiz et al. ${ }^{[43]}$ and by Yao et al. ${ }^{[44]}$ Microwave irradiation (MWI) induced heating in solvents has been used as a rapid way to synthesize graphene sheets. Due to the difference in the solvent and reactant dielectric constants, selective dielectric heating of the reactants by microwave causes an instantaneous internal temperature rise and thereby reduction of GO. ${ }^{[45]}$

\subsubsection{Arc Discharge}

Arc evaporation of graphite in presence of hydrogen yields high-quality graphene (HG). ${ }^{[6]}$ The graphene sheets with mostly 2-3 layers have a flake size of 100-200 nm. The presence of $\mathrm{H}_{2}$ during the arc-discharge process terminates the dangling carbon bonds with hydrogen and prevents the formation of closed structures like carbon nanotubes. The conditions that are favorable for obtaining graphene in the inner walls are the high current (above $100 \mathrm{~A})$, the high voltage ( $>50 \mathrm{~V})$, and the high pressure of hydrogen (above 200 torr). Figs 6a-c show TEM and AFM images and height profile of the HG sample respectively. Arc-discharge in a helium atmosphere also yields graphene sheets with the number of layers depending on the pressure and current. ${ }^{[46]}$ Hydrogen arc-discharge has been conveniently employed to dope graphene with boron and nitrogen. ${ }^{[47]}$ $\mathrm{H}_{2}$ +diborane and $\mathrm{H}_{2}$ +pyridine (or ammonia) are used instead of pure $\mathrm{H}_{2}$ to dope with boron and nitrogen respectively. Figs

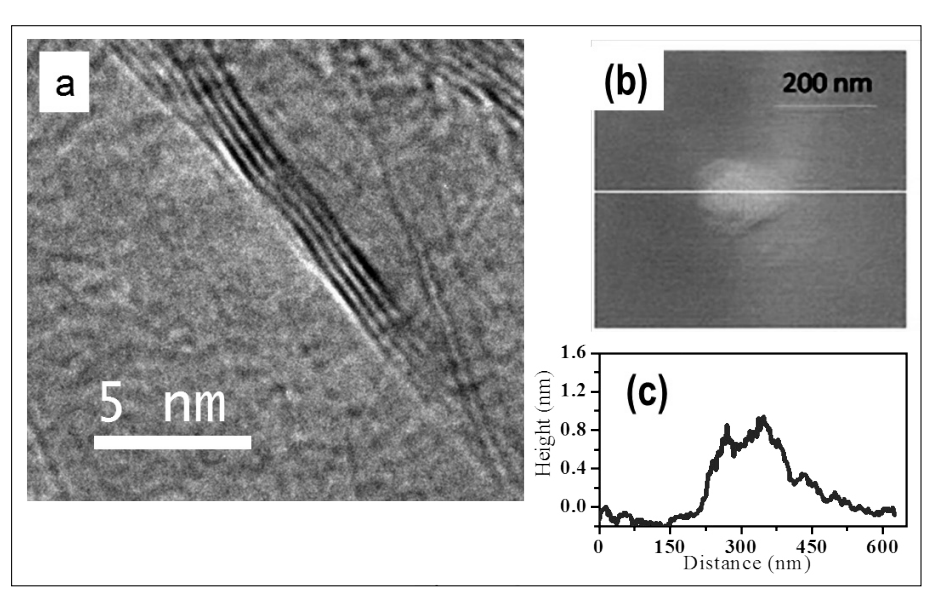

Fig. 6. (a) TEM and (b) AFM images of HG prepared by arc discharge of graphite in hydrogen. (c) The height profile of AFM image in (b) (reprinted with permission from ref. [6]. Copyright American Chemical Society, 2009). 


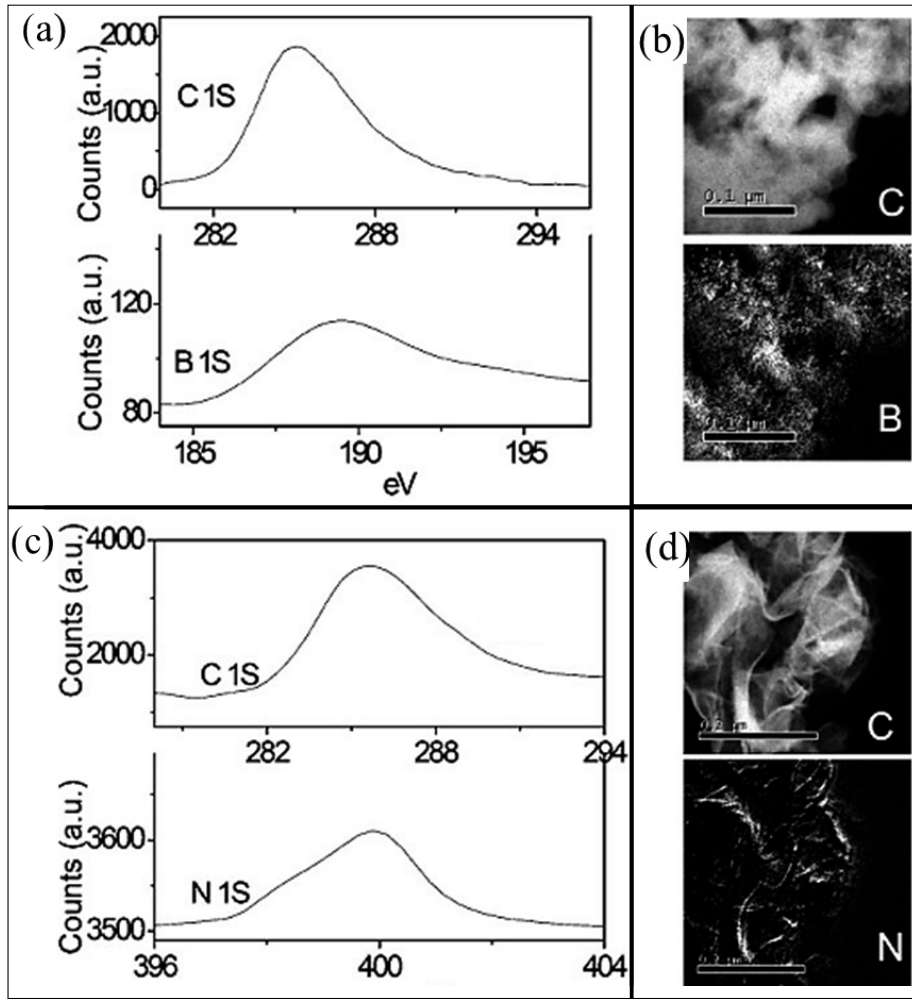

Fig. 7. (a) C 1s and B 1s XPS and (b) EELS elemental mapping of B-doped arc discharge graphene, (c) C 1s and N 1s XPS and (d) EELS elemental mapping of $\mathrm{N}$-doped graphene arc discharge graphene (reprinted with permission from ref. [48]. Copyright 2009 WILEY-VCH Verlag $\mathrm{GmbH} \& \mathrm{Co}$. KGaA, Weinheim).

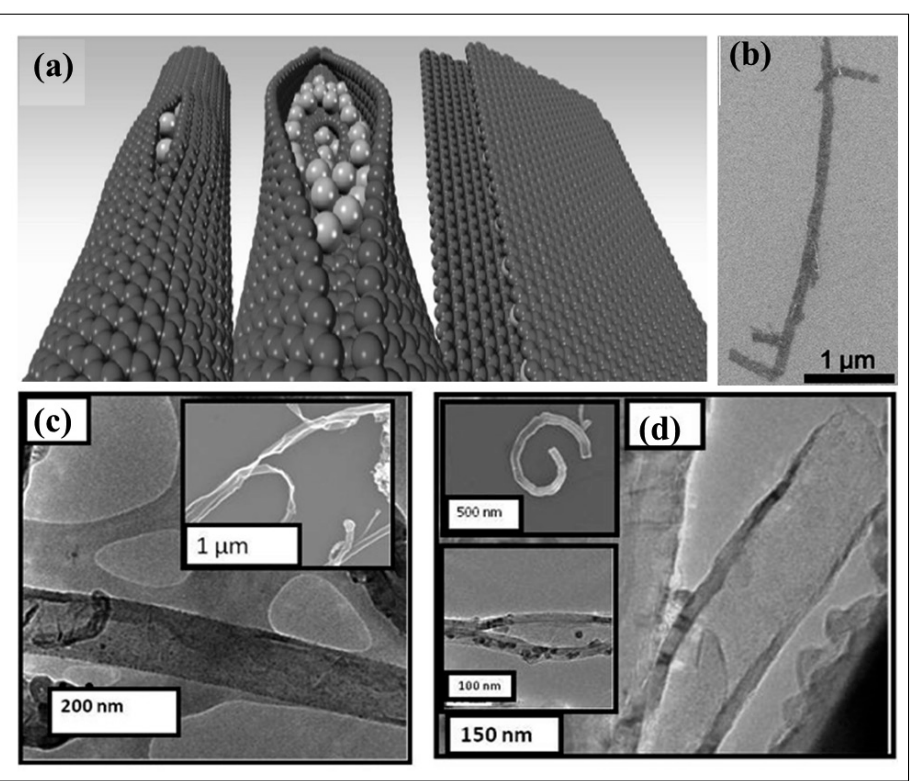

Fig. 8. (a) Schematic of the splitting process involving potassium intercalation between the nanotube walls and their longitudinal unzipping to from GNRs, (b) TEM image of a GNR obtained from K-intercalation induced unzipping (reprinted with permission from ref. [59]. Copyright American Chemical Society, 2011), (c) N-doped GNRs obtained from laser induced unzipping of N-doped MWNTs. Inset shows the corresponding FESEM image and (d) B-doped GNRs prepared similarly from B-doped MWNTs. Top inset shows the corresponding FESEM image. Bottom inset shows a TEM image of partially opened B-MWNT (reprinted with permission from ref. [8]. Copyright Royal Society of Chemistry, 2011).
$7 \mathrm{a}$ and $\mathrm{b}$ show the typical core level X-ray photoelectron spectra (XPS) and electron energy loss spectroscopy (EELS) map of boron-doped graphene respectively. The peak positions of $\mathrm{C} 1 \mathrm{~s}$ and $\mathrm{B} 1 \mathrm{~s}$ indicate that boron is bonded to the $\mathrm{sp}^{2}$ carbon. The EELS map shows homogenous distribution of boron. XPS and EELS analysis give boron contents of 3.1 at $\%$ and 2.4 at $\%$ respectively. Figs 7c and d show XPS and EELS map of nitrogen-doped graphene respectively. Deconvolution of nitrogen core level XPS gives two peaks $397.3 \mathrm{eV}$ and $400 \mathrm{eV}$ corresponding to pyridine- and pyrimidine-type nitrogen. Nitrogen content is found to be 1.4 at $\%$ and 0.9 at $\%$ from XPS and EELS analysis respectively. ${ }^{[48]}$

Graphene sheets have also been prepared from GO using a hydrogen arc discharge process as a rapid heating method. [49] Graphene nanosheets can also be prepared by arc discharge in air with the yield depending strongly on the initial air pressure. ${ }^{[50]}$ Graphene nanosheets so obtained are 100-200 nm wide with mainly two-layers. Annealing of nanodiamond at $1650{ }^{\circ} \mathrm{C}$ or higher temperature in $\mathrm{He}$ atmosphere also yields few-layer graphene.[2b,12]

\subsection{Chemical Methods}

Electrochemical reduction has been employed as a convenient method to synthesize graphene on large scale. ${ }^{[51]}$ The reduction initiates at $-0.8 \mathrm{~V}$ and is completed by $-1.5 \mathrm{~V}$ with the formation of a black precipitate of graphene on the bare graphite electrode. A combination of coupled electrochemical reduction with a spray coating technique has been employed to prepare large-area and patterned RGO films on various conductive and insulating substrates. ${ }^{[52]}$ Organic dispersions of graphene oxide in polar organic solvents yields few-layer graphene under reflux conditions to afford electrically conductive, chemically active reduced graphene oxide (CARGO) with tunable C/O ratios, dependent on the boiling point of the solvent. The dispersing medium must have a boiling point above $150{ }^{\circ} \mathrm{C}$ (the initiation point of the mass loss feature in the TGA profile of graphene oxide) and be able to disperse both graphene oxide and CARGO for example DMF, dimethyl sulfoxide and $\mathrm{N}$-methylpyrrolidone have been used for the purpose. ${ }^{[51 \mathrm{c}]}$

Intercalation of alkali metal ions and exfoliation yields few-layer graphene in high quantities. Thus $\mathrm{K}, \mathrm{Cs}$ and $\mathrm{NaK}_{2}$ intercalated graphite were prepared by reacting alkali metals with acid-intercalated exfoliated graphite in pyrex sealed tubes. ${ }^{[53]}$ These intercalated carbons were then treated with ethanol causing a vigorous reaction and exfoliation. Stable high-concentration suspensions of few layer graphene are also obtained by direct sonication in ionic liquids. ${ }^{[54]}$

\section{Graphene Nanoribbons}

Graphene nanoribbons (GNRs) are quasi one-dimensional graphene nanostructures and can be thought of as thin strips of graphene or unrolled carbon nanotubes. Graphene nanoribbons have attracted attention because of their novel electronic and spin transport properties. ${ }^{[55]}$ Longitudinal unzipping of nanotubes is the most commonly used method to GNRs. Kosynkin et al.[7] used strong oxidizing agents like the $\mathrm{KMnO}_{4}-\mathrm{H}_{2} \mathrm{SO}_{4}$ mixture to oxidize and induce defects in the carbon nanotubes which then underwent unzipping to produce nanoribbons. Higginbotham et al. ${ }^{[56]}$ used a second acid like trifluroacetic acid or $\mathrm{H}_{3} \mathrm{PO}_{4}$ to control the oxidation. This allowed the protection of the vicinal diols formed on the basal plane of graphene during the oxidation, thereby preventing over oxidation to diones and subsequent hole generation. The GNRs thus produced have less defects or holes. Jiao et al. ${ }^{[57]}$ carried out mild gas phase oxidation to create defects on CNTs. A dispersion of these CNTs in 1,2-dichloroethane (DCE) solution of poly(m-phenylenevinyleneco-2,5-dioctoxy-p-phenylenevinylene) (PmPV) by sonication yield high quality unzipped nanoribbons. Cano-Marquez et al..$^{[58]}$ obtained GNRs after treatment of CNTs with $\mathrm{Li}$ in liquid $\mathrm{NH}_{3}$ followed by treatment with $\mathrm{HCl}$. Kosynkin et al.[59] 


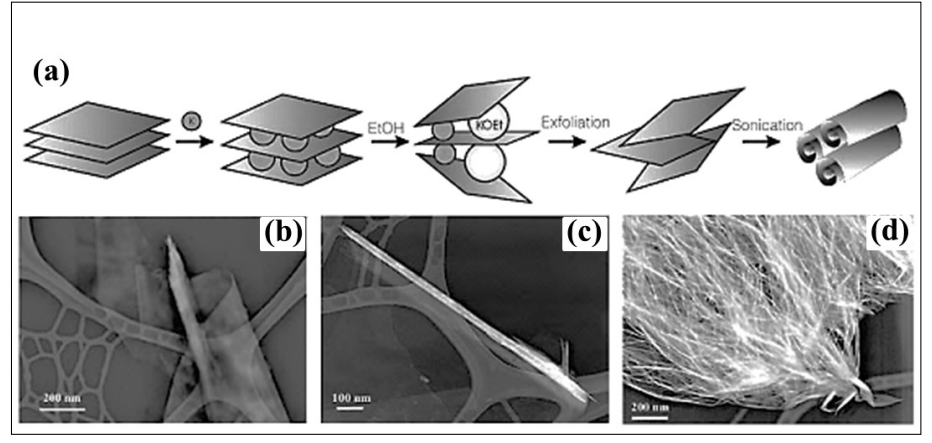

Fig. 9. (a) Schematic of the process of potassium metal intercalation and then exfoliation with ethanol followed by sonication to produce CNS,

TEM images of (b) a partially formed nanoscroll, (c) an open ended nanoscroll and (d) a mass of scrolled material. (reprinted with permission from ref. [69]. Copyright American Association for the Advancement of Science, 2003)

unzipped CNTs by K-intercalation using potassium vapour and further sonication in chlorosuphonic acid. The nanoribbons obtained by this method have fewer defects compared to those prepared by oxidative unzipping. Fig. 8a shows a schematic of potassium intercalation and sequential longitudinal splitting of the CNT walls to yield a nanoribbon stack. TEM image of one such GNR is shown in Fig. 8b.

Doped and undoped MWNTs have been unzipped using excimer laser irradiation in solvents like DMF. The power of laser to be used depends on the nature of MWNTs taken. Nitrogen and boron doped MWNTs were readily prepared by this method. ${ }^{[8]}$ Thus synthesized GNRs were free of any oxygen impurities or functional groups as is evident from the Raman spectrum. The GNRs had widths in the range 60-70 nm with lengths up to a few $\mu \mathrm{m}$. Figs $8 \mathrm{c}$ and $d$ show the TEM and FESEM image of laser unzipped nitrogen and boron doped nanoribbons, respectively. Longitudinal cutting of MWNTs using plasma also yields graphene nanoribbons. Jiao et al. ${ }^{[60]} \mathrm{em}-$ bedded the MWNTs in PMMA and then carried out controlled unzipping by $\mathrm{Ar}$ plasma etching. Oxygen plasma etching of graphene sheets on the other hand yielded graphene nanoribbons of width 10-100 nm and length $1-2 \mu \mathrm{m} .{ }^{[61]}$

Lithographic patterning of graphene is another way to prepare narrow GNRs. Hydrogen silsesquioxane, a negative tone e-beam resist, is used to protect the underlying graphene layer. The unprotected layer gets etched away by the oxygen plasma. ${ }^{[61]}$ Geometrically and crystallographically oriented GNRs were etched from graphene sheets by applying constant bias potential to the STM tip (higher than that used for imaging). [62]

All the above methods produced GNRs of width more than $20 \mathrm{~nm}$ with edge roughness of $\sim 5 \mathrm{~nm}$. Li et al. ${ }^{[55 \mathrm{a}]}$ for the first time prepared sub- $10 \mathrm{~nm}$ width GNRs chemically. They sonicated the thermally exfoliated

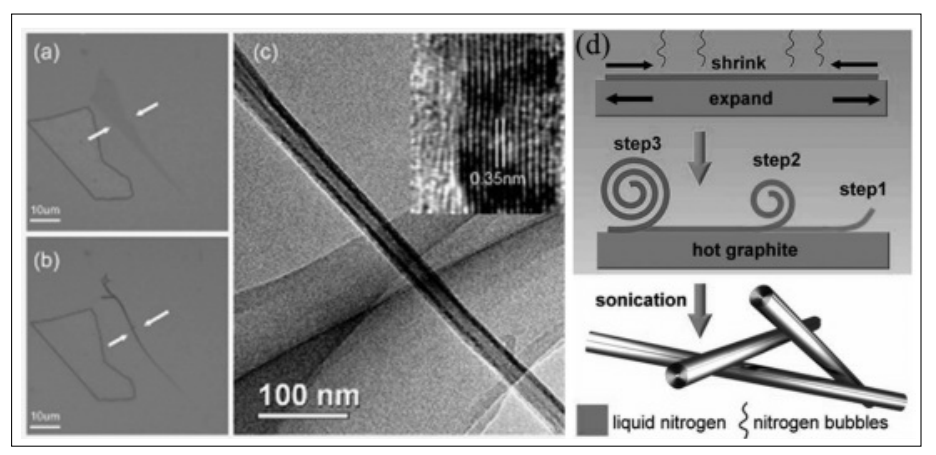

Fig. 10. Optical microscopic image of (a) original graphene sheet on $\mathrm{SiO}_{2}$ and (b) the nanoscroll obtained from it by sonication in isopropanol, (c) TEM image of the nanoscroll. Inset shows the high resolution TEM image of the nanoscroll with interlayer spacing of $0.35 \mathrm{~nm}$, (reprinted with permission from ref. [21]. Copyright American Chemical Society, 2009) and (d) Schematic illustration of the exfoliation and rolling up process involving treatment of graphite dispersed in liquid nitrogen to microwave assisted spark. (reprinted with permission from ref. [71]. Copyright 2011 WILEY-VCH Verlag GmbH \& Co. KGaA, Weinheim)

graphite by dispersing it in a 1, 2-dichloroethane (DCE) solution of poly(m-phenylenevinylene-co-2,5-dioctoxy-p-phenylenevinylene) (PmPV) and removing the larger pieces by centrifugation. GNRs have also been obtained by sonochemical cutting of graphene sheets, involving oxygeninduced unzipping of graphene sheets. [63] GNRs are also obtained by plasma-enhanced chemical vapor deposition on $\mathrm{Pd}$ nanowire templates. On simply removing the Pd nanowires, a tubular-shaped collapsed graphene with closed edge is obtained. On selectively etching the graphene layers on the top part of the metal nanowire by $\mathrm{O}_{2}$ plasma yields GNRs.[64] Wang et al. ${ }^{[65]}$ have prepared 20-30-nm-wide graphene nanoribbon arrays lithographically, and used gas phase etching chemistry to narrow the ribbons down to $<10 \mathrm{~nm}$, thereby achieving a high on/off ratio up to $\sim 10^{4}$. Precise control over topologies and widths of GNRs can only be obtained by bottom-up fabrication. Molecular precursors are first coupled on substrates to form linear polyphenylenes. These are subsequently cyclo-dehydrogenated to prepare GNRs with predefined edge structure and morphology. ${ }^{[66]}$ Suzuki-Miyaura polymerization of the bis-boronic esters with diiodobenzenes yields polyphenylenes resembling GNRs.[67]

\section{Graphene Nano-scrolls}

Graphene nano-scrolls or as they are more popularly known carbon nano-scrolls (CNS) were first described by Bacon et al. ${ }^{[68]}$ in as early as 1960 (termed then as graphite whiskers). First chemical synthesis of carbon-nanoscrolls were carried out by Viculis et al. ${ }^{[69]}$ by first intercalating graphite heavily with potassium metal in pyrex sealed tubes to yield the graphite intercalated compound, $\mathrm{KC}_{8}$. On dispersing $\mathrm{KC}_{8}$ in ethanol by sonication, graphite undergoes exfoliation and simultaneously rolling up to yield scrolls. Fig. 9a shows a schematic representation of the formation of nanoscrolls from graphite. CNS prepared by this method had an average diameter of $40 \mathrm{~nm}$ and a very high surface area of $2630 \mathrm{~m}^{2} / \mathrm{g}$. Figs $9 \mathrm{~b}$ and $\mathrm{c}$ show TEM image of an individual nano-scroll with a partially formed scroll and an open ended scroll respectively. An image of bulk CNS is shown in Fig. 9d. CNS has also been prepared from acceptor-type graphite intercalation compounds. Graphite nitrate prepared from natural graphite using $\mathrm{HNO}_{3}$ and ozone was sonicated in ethanol to yield CNS. [70] The above methods use chemical intercalation and are bound to introduce impurities.

High-quality graphene scrolls have been prepared by using iso-propanol to induce rolling of predefined thin graphene flakes on $\mathrm{SiO}_{2} / \mathrm{Si}$ substrates. ${ }^{[21]}$ This is accomplished by depositing thin graphene sheets from micromechanical exfoliation onto $\mathrm{SiO}_{2} / \mathrm{Si}$ substrates followed by sonication in iso-propanol. The alcohol enters the spaces between the graphene and substrate and thereby lifts it up from the surface. Due to perturbation by sonication the free graphene ends curve up followed by scrolling up to result in tightly packed scrolls with an interlayer spacing of 0.35 $\mathrm{nm}$. Figs 10a and b show optical images of original graphene flakes and scrolls on $\mathrm{SiO}_{2}$ respectively. The TEM image of the CNS shows tightly packed nanoscroll (Fig. 10c).

Microwave sparks have been used to provide thermal shock to induce exfoliation and scrolling.[71] Graphite flakes are immersed in liquid nitrogen while irradiating with microwave. The flakes experienced rapid heating and expansion while 


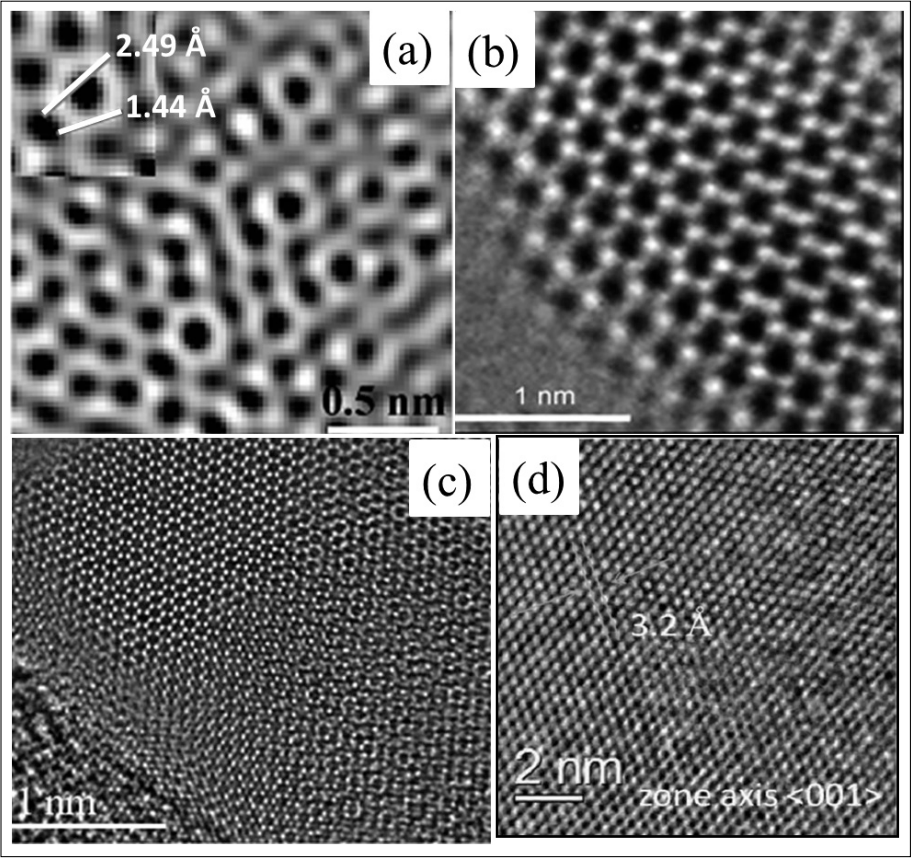

Fig. 11. Highresolution TEM images of few-layered (a) BN, (b) $\mathrm{MoS}_{2}$, (c) $\mathrm{MoSe}_{2}$ and (d) single-layered GaSe (reprinted with permission from ref. [77], Copyright American Chemical Society, 2010; ref. [76a], Copyright 2011 WILEY-VCH Verlag GmbH \& Co. KGaA, Weinheim; ref. [76b], Copyright Royal Society of Chemistry, 2011; ref. [78], Copyright 2012 WILEY-VCH Verlag $\mathrm{GmbH} \& \mathrm{Co}$. KGaA, Weinheim).

the surface of each flake cooled by liquid $\mathrm{N}_{2}$ underwent contraction. The large strain differences between the surface and bulk resulted in scrolling up of the sheets (see Fig. 10d for a schematic of the formation of nanoscrolls by this method).

\section{Inorganic Graphene Analogues}

Various layered inorganic compounds are known, the most well-known being $\mathrm{BN}, \mathrm{MoS}_{2}, \mathrm{WS}_{2}$ etc. Fullerene-type structures of these layered inorganic materials were made by Tenne and co-workers. ${ }^{[72]}$ Nanotubes soon followed suite. ${ }^{[73]}$ Graphene analogues of these materials with few-layers have recently been prepared and characterized. [74] Single-layer as well as few-layer graphene-like species of $\mathrm{MoS}_{2}$ and other metal chalcogenides have been prepared by both physical and chemical methods. Physical methods are micromechanical cleavage and exfoliation by ultrasonication in solvents. ${ }^{[75]}$ Chemical methods mostly include thermal decomposition of mixture of metal precursors with thiourea, solvothermal synthesis and $\mathrm{Li}$ metal intercalation followed by exfoliation. ${ }^{[76]}$ Few-layered $\mathrm{BN}$ has been prepared by high-temperature reaction of boric acid and urea at high temperature. ${ }^{[77]}$ It is possible to vary the number of layers by varying the ratio of boric acid and urea. BN can also be prepared by laser exfoliation of bulk powder in DMF or by ultrasonication in polar solvents. Figs $11 \mathrm{a}$ and $11 \mathrm{~b}$ show typical TEM images of 2-4 layered BN and $\mathrm{MoS}_{2}$ prepared by high-temperature decomposition of precursors. A high-resolution TEM image of single-layered $\mathrm{MoS}_{2}$ with atomic resolution is shown in Fig. 11c. Micromechanical cleavage also readily yields single- and few-layered GaS and
GaSe. ${ }^{[78]}$ A high-resolution TEM image of single-layered GaSe is shown in Fig. 11d. Ultrasonication in solvents has become a routine method to obtain few-layered species of metal organic frameworks.

\section{Conclusions}

It is indeed gratifying that graphene which was once considered to be difficult to prepare has been prepared by physical as well as chemical methods. Although physical methods such as micromechanical exfoliation and ultrasonication are easily employed, chemical methods have provided ways of preparing both singleand few-layer graphene in larger quantities. Chemical methods have also been useful in generating graphene-like species of other inorganic layered materials such as $\mathrm{MoS}_{2}$ and $\mathrm{BN}$, besides ultrasonication and micromechanical cleavage. It is noteworthy that nanoscrolls of graphene can be generated by intercalation of graphite with potassium. It would not be surprising that graphene-like species of a variety of layered inorganic materials involving metal oxides will be prepared in the next few years.

Received: October 10, 2012

[1] K. S. Novoselov, A. K. Geim, S. V. Morozov, D. Jiang, Y. Zhang, S. V. Dubonos, I. V. Grigorieva, A. A. Firsov, Science 2004, 306, 666.

[2] a) C. N. R. Rao, H. S. S. R. Matte, K. S. Subrahmanyam, Acc. Chem. Res. 2012, doi: 10.1021/ar300033m; b) C. N. R. Rao, K. S. Subrahmanyam, H. S. S. Ramakrishna Matte, B. Abdulhakeem, A. Govindaraj, B. Das, P. Kumar, A. Ghosh, D. J. Late, Sci. Tech. Adv. Mater. 2010, 11, 054502 .

[3] a) D. C. Marcano, D. V. Kosynkin, J. M. Berlin, A. Sinitskii, Z. Sun, A. Slesarev, L. B. Alemany, W. Lu, J. M. Tour, ACS Nano 2010, 4, 4806; b) S. Park, J. An, I. Jung, R. D. Piner, S. J. An, X. Li, A. Velamakanni, R. S. Ruoff, Nano Lett. 2009, 9, 1593; c) W. S. Hummers, R. E. Offeman, J. Am. Chem. Soc. 1958, 80, 1339; d) M. J. Allen, V. C. Tung, R. B. Kaner, Chem. Rev. 2009, 110, 132; e) V. C. Tung, M. J. Allen, Y. Yang, R. B. Kaner, Nat. Nanotechnol. 2009, 4 25; f) J. I. Paredes, S. Villar-Rodil, A. MartinezAlonso, J. M. D. Tascon, Langmuir 2008, 24 , 10560.

[4] C. Valles, C. Drummond, H. Saadaoui, C. A. Furtado, M. He, O. Roubeau, L. Ortolani, M. Monthioux, A. Penicaud, J. Am. Chem. Soc. 2008, 130, 15802 .

[5] a) H. C. Schniepp, J.-L. Li, M. J. McAllister, H. Sai, M. Herrera-Alonso, D. H. Adamson, R. K. Prud'homme, R. Car, D. A. Saville, I. A. Aksay, J. Phys. Chem. B 2006, 110, 8535; b) M. J. McAllister, J.-L. Li, D. H. Adamson, H. C. Schniepp, A. A. Abdala, J. Liu, M. Herrera-Alonso, D. L. Milius, R. Car, R. K. Prud'homme, I. A. Aksay, Chem. Mater. 2007, 19, 4396.

[6] K. S. Subrahmanyam, L. S. Panchakarla, A. Govindaraj, C. N. R. Rao, J. Phys. Chem. C 2009, 113, 4257

[7] D. V. Kosynkin, A. L. Higginbotham, A. Sinitskii, J. R. Lomeda, A. Dimiev, B. K. Price, J. M. Tour, Nature 2009, 458, 872.

[8] P. Kumar, L. S. Panchakarla, C. N. R. Rao, Nanoscale 2011, 3, 2127.

[9] K. S. Novoselov, A. K. Geim, S. V. Morozov, D. Jiang, M. I. Katsnelson, I. V. Grigorieva, S. V. Dubonos, A. A. Firsov, Nature 2005, 438, 197.

[10] a) H. Hiura, T. W. Ebbesen, J. Fujita, K. Tanigaki, T. Takada, Nature 1994, 367, 148; b) T. W. Ebbesen, H. Hiura, Adv. Mater 1995 , 7,582 ; c) T. M. Bernhardt, B. Kaiser, K. Rademann, Surf. Sci. 1998, 408, 86; d) H. Roy, J. Appl. Phys. 1998, 83, 4695; e) X. Lu, M. Yu, H. Huang, R. S. Ruoff, Nanotechnol. 1999, 10, $269 ;$ f) H. V. Roy, C. Kallinger, K. Sattler, Surf. Sci. 1998, 407, 1

[11] D. S. L. Abergel, A. Russell, V. I. Falko, Appl. Phys. Lett. 2007, 91, 063125.

[12] C. N. R. Rao, A. K. Sood, K. S. Subrahmanyam, A. Govindaraj, Angew. Chem. Int. Ed. 2009, 48, 7752.

[13] A. K. Geim, K. S. Novoselov, Nat. Mater. 2007, 6,183 .

[14] Z. H. Ni, H. M. Wang, J. Kasim, H. M. Fan, T. Yu, Y. H. Wu, Y. P. Feng, Z. X. Shen, Nano Lett. 2007, 7, 2758

[15] Y. Hernandez, V. Nicolosi, M. Lotya, F. M. Blighe, Z. Sun, S. De, I. T. McGovern, B. Holland, M. Byrne, Y. K. GunKo, J. J. Boland, P. Niraj, G. Duesberg, S. Krishnamurthy, R. Goodhue, J. Hutchison, V. Scardaci, A. C. Ferrari, J. N. Coleman, Nat. Nanotechnol. 2008, 3,563 .

[16] A. B. Bourlinos, V. Georgakilas, R. Zboril, T. A. Steriotis, A. K. Stubos, Small 2009, 5, 1841.

[17] C. E. Hamilton, J. R. Lomeda, Z. Sun, J. M. Tour, A. R. Barron, Nano. Lett. 2009, 9, 3460.

[18] A. ONeill, U. Khan, P. N. Nirmalraj, J. Boland, J. N. Coleman, J. Phys. Chem. C 2011, 115, 5422.

[19] Y. Hernandez, M. Lotya, D. Rickard, S. D. Bergin, J. N. Coleman, Langmuir 2009, 26, 3208.

[20] D. R. Dreyer, S. Park, C. W. Bielawski, R. S. Ruoff, Chem. Soc. Rev. 2010, 39, 228.

[21] X. Xie, L. Ju, X. Feng, Y. Sun, R. Zhou, K. Liu, S. Fan, Q. Li, K. Jiang, Nano Letters 2009, 9, 2565.

[22] S. Stankovich, D. A. Dikin, R. D. Piner, K. A. Kohlhaas, A. Kleinhammes, Y. Jia, Y. Wu, S. T. Nguyen, R. S. Ruoff, Carbon 2007, 45, 1558.

[23] V. H. Pham, T. V. Cuong, T.-D. Nguyen-Phan, H. D. Pham, E. J. Kim, S. H. Hur, E. W. Shin, S. Kim, J. S. Chung, Chem. Comm. 2010, 46, 4375 . 
[24] X. Zhou, J. Zhang, H. Wu, H. Yang, J. Zhang, S. Guo, J. Phys. Chem. C 2011, 115, 11957.

[25] C. Zhu, S. Guo, Y. Fang, S. Dong, ACS Nano 2010, 4, 2429.

[26] J. Zhang, H. Yang, G. Shen, P. Cheng, J. Zhang, S. Guo, Chem. Commun. 2010, 46, 1112.

[27] G. Wang, J. Yang, J. Park, X. Gou, B. Wang, H. Liu, J. Yao, J. Phys. Chem. 2008, 112, 8192.

[28] X. Fan, W. Peng, Y. Li, X. Li, S. Wang, G. Zhang, F. Zhang, Adv. Mater. 2008, 20, 4490.

[29] C. A. Amarnath, C. E. Hong, N. H. Kim, B.-C. Ku, T. Kuila, J. H. Lee, Carbon 2011, 49, 3497.

[30] P. Kumar, B. Das, B. Chitara, K. S. Subrahmanyam, K. Gopalakrishnan, S. B. Krupanidhi, C. N. R. Rao, Macromol. Chem. Phys. 2012, 213, 1146.

[31] P. Kumar, K. S. Subrahmanyam, C. N. R. Rao, Mater. Exp. 2011, 1, 252.

[32] K. S. Kim, Y. Zhao, H. Jang, S. Y. Lee, J. M. Kim, K. S. Kim, J.-H. Ahn, P. Kim, J.-Y. Choi, B. H. Hong, Nature 2009, 457, 706.

[33] S.-Y. Kwon, C. V. Ciobanu, V. Petrova, V. B. Shenoy, J. Bareno, V. Gambin, I. Petrov, S. Kodambaka, Nano Lett. 2009, 9, 3985.

[34] P. W. Sutter, J.-I. Flege, E. A. Sutter, Nat. Mater. 2008, 7, 406

[35] J. Coraux, A. T. N`Diaye, C. Busse, T. Michely, Nano Lett. 2008, 8, 565.

[36] X. Li, W. Cai, J. An, S. Kim, J. Nah, D. Yang, R. Piner, A. Velamakanni, I. Jung, E. Tutuc, S. K. Banerjee, L. Colombo, R. S. Ruoff, Science 2009, 324, 1312.

[37] A. Reina, X. Jia, J. Ho, D. Nezich, H. Son, V. Bulovic, M. S. Dresselhaus, J. Kong, Nano. Lett. 2008, 9, 30

[38] a) J. J. Wang, M. Y. Zhu, R. A. Outlaw, X. Zhao, D. M. Manos, B. C. Holloway, V. P. Mammana, Appl. Phys. Lett. 2004, 85, 1265; b) J. Wang, M. Zhu, R. A. Outlaw, X. Zhao, D. M. Manos, B. C. Holloway, Carbon 2004, 42, 2867; c) M. Zhu, J. Wang, B. C. Holloway, R. A. Outlaw, X. Zhao, K. Hou, V. Shutthanandan, D. M. Manos, Carbon 2007, 45, 2229.

[39] M. Choucair, P. Thordarson, J. A. Stride, Nat. Nanotechnol. 2009, 4, 30

[40] A. Ghosh, K. V. Rao, S. J. George, C. N. R. Rao, Chem.-Eur. J. 2010, 16, 2700.

[41] X. Li, G. Zhang, X. Bai, X. Sun, X. Wang, E. Wang, H. Dai, Nat. Nanotechnol. 2008, 3, 538.

[42] X. Guoqing, H. Wontae, K. Namhun, M. C. Sung, C. Heeyeop, Nanotechnol. 2010, 21, 405201 .
[43] O. O. Ekiz, M. Urel, H. Guner, A. K. Mizrak, A. Dana, ACS Nano 2011, 5, 2475.

[44] P. Yao, P. Chen, L. Jiang, H. Zhao, H. Zhu, D. Zhou, W. Hu, B.-H. Han, M. Liu, Adv. Mater. 2010, 22, 5008

[45] H. M. A. Hassan, V. Abdelsayed, A. E. R. S. Khder, K. M. AbouZeid, J. Terner, M. S. ElShall, S. I. Al-Resayes, A. A. El-Azhary, J. Mater. Chem. 2009, 19, 3832.

[46] C. Wu, G. Dong, L. Guan, Physica E 2010, 42, 1267.

[47] L. S. Panchakarla, A. Govindaraj, C. N. R. Rao, Inorg. Chim. Acta 2009, 363, 4163.

[48] L. S. Panchakarla, K. S. Subrahmanyam, S. K. Saha, A. Govindaraj, H. R. Krishnamurthy, U. V. Waghmare, C. N. R. Rao, Adv. Mater. 2009, 21, 4726 .

[49] Z.-S. Wu, W. Ren, L. Gao, J. Zhao, Z. Chen, B. Liu, D. Tang, B. Yu, C. Jiang, H.-M. Cheng, ACS Nano 2009, 3, 411 .

[50] W. Zhiyong, L. Nan, S. Zujin, G. Zhennan, Nanotechnol. 2010, 21, 175602.

[51] a) H.-L. Guo, X.-F. Wang, Q.-Y. Qian, F.-B. Wang, X.-H. Xia, ACS Nano 2009, 3, 2653; b) R. S. Sundaram, C. Gómez-Navarro, K. Balasubramanian, M. Burghard, K. Kern, $A d v$. Mater. 2008, 20, 3050; c) O. C. Compton, B. Jain, D. A. Dikin, A. Abouimrane, K. Amine, S. T. Nguyen, ACS Nano 2011, 5, 4380.

[52] M. Zhou, Y. Wang, Y. Zhai, J. Zhai, W. Ren, F. Wang, S. Dong, Chem.-Eur. J. 2009, 15, 6116.

[53] L. M. Viculis, J. J. Mack, O. M. Mayer, H. T. Hahn, R. B. Kaner, J. Mater. Chem. 2005, 15, 974.

[54] X. Wang, P. F. Fulvio, G. A. Baker, G. M. Veith, R. R. Unocic, S. M. Mahurin, M. Chi, S. Dai, Chem. Comm. 2010, 46, 4487.

[55] a) X. Li, X. Wang, L. Zhang, S. Lee, H. Dai, Science 2008, 319, 1229; b) X. Wang, Y. Ouyang, X. Li, H. Wang, J. Guo, H. Dai, Phys. Rev. Lett. 2008, 100, 206803; c) M. Y. Han, Ouml, B. zyilmaz, Y. Zhang, P. Kim, Phys. Rev. Lett. 2007, 98, 206805; d) A. Cresti, N. Nemec, B. Biel, G. Niebler, F. o. Triozon, G. Cuniberti, S. Roche, Nano Res. 2008, 1,361 .

[56] A. L. Higginbotham, D. V. Kosynkin, A. Sinitskii, Z. Sun, J. M. Tour, ACS Nano 2010, 4, 2059.

[57] L. Jiao, X. Wang, G. Diankov, H. Wang, H. Dai, Nat. Nanotechnol. 2011, 6, 132.

[58] A. G. Cano-Marquez, F. J. Rodrguez-Macias, J. Campos-Delgado, C. G. Espinosa-Gonzalez, F. Tristan-Lopez, D. Ramrez-Gonzalez, D. A. Cullen, D. J. Smith, M. Terrones, Y. I. VegaCantu, Nano Lett. 2009, 9, 1527.
[59] D. V. Kosynkin, W. Lu, A. Sinitskii, G. Pera, Z. Sun, J. M. Tour, ACS Nano 2011, 5, 968

[60] L. Jiao, L. Zhang, X. Wang, G. Diankov, H. Dai, Nature 2009, 458, 877.

[61] Z. Chen, Y.-M. Lin, M. J. Rooks, P. Avouris, Physica E 2007, 40, 228.

[62] L. Tapaszto, G. Dobrik, P. Lambin, L. P. Biro, Nat. Nanotechnol. 2008, 3, 397.

[63] Z.-S. Wu, W. Ren, L. Gao, B. Liu, J. Zhao, H.-M. Cheng, Nano Res. 2010, 3, 16.

[64] W. J. Yu, S. H. Chae, D. Perello, S. Y. Lee, G. H. Han, M. Yun, Y. H. Lee, ACS Nano 2010, 4, 5480.

[65] X. Wang, H. Dai, Nat. Chem. 2010, 2, 661.

[66] J. Cai, P. Ruffieux, R. Jaafar, M. Bieri, T. Braun, S. Blankenburg, M. Muoth, A. P. Seitsonen, M. Saleh, X. Feng, K. Mullen, R. Fasel, Nature 2010, 466, 470.

[67] X. Yang, X. Dou, A. Rouhanipour, L. Zhi, H. J. Rader, K. Mullen, J. Am. Chem. Soc. 2008, 130, 4216.

[68] R. Bacon, J. Appl. Phys. 1960, 31, 283.

[69] L. M. Viculis, J. J. Mack, R. B. Kaner, Science 2003, 299, 1361.

[70] M. V. Savoskin, V. N. Mochalin, A. P. Yaroshenko, N. I. Lazareva, T. E. Konstantinova, I. V. Barsukov, I. G. Prokofiev, Carbon 2007, 45, 2797.

[71] J. Zheng, H. Liu, B. Wu, Y. Guo, T. Wu, G. Yu, Y. Liu, D. Zhu, Adv. Mater. 2011, 23, 2460.

[72] R. Tenne, Nat. Nanotechnol. 2006, 1, 103.

[73] C. N. R. Rao, A. Govindaraj, Adv. Mater. 2009, 21, 4208.

[74] Y. Gao, X. Chen, H. Xu, Y. Zou, R. Gu, M. Xu, A. K. Y. Jen, H. Chen, Carbon 2010, 48, 4475.

[75] a) K. F. Mak, C. Lee, J. Hone, J. Shan, T. F. Heinz, Phys. Rev. Lett. 2010, 105, 136805; b) G. Cunningham, M. Lotya, C. S. Cucinotta, S. Sanvito, S. D. Bergin, R. Menzel, M. S. P. Shaffer, J. N. Coleman, ACS Nano 2012, 6, 3468.

[76] a) H. S. S. Ramakrishna Matte, A. Gomathi, A. K. Manna, D. J. Late, R. Datta, S. K. Pati, C. N. R. Rao, Angew. Chem. Int. Ed. 2010, 49, 4059; b) H. S. S. R. Matte, B. Plowman, R. Datta, C. N. R. Rao, Dalton Trans. 2011, 40, 10322.

[77] A. Nag, K. Raidongia, K. P. S. S. Hembram, R. Datta, U. V. Waghmare, C. N. R. Rao, ACS Nano 2010, 4, 1539.

[78] D. J. Late, B. Liu, J. Luo, A. Yan, H. S. S. R. Matte, M. Grayson, C. N. R. Rao, V. P. Dravid, Adv. Mater. 2012, 24, 3549. 